\title{
An Improved Method for Automatic Segmentation and Accurate Detection of Brain Tumor in Multimodal MRI
}

\author{
K Bhima \\ Associate Professor, BVRIT, Narsapur, Telangana State, India \\ Email: bhima.mnnit@gmail.com \\ Dr A Jagan \\ Professor and HOD-CSE, BVRIT, Narsapur, Telangana State, India \\ Email: jagan.amgoth@bvrit.ac.in
}

\begin{abstract}
Automatic segmentation and detection of brain tumor is a notoriously complicated issue in Magnetic Resonance Image. The similar state-of-art segmentation methods and techniques are limited for the detection of tumor in multimodal brain MRI. Thus this work deals about the accurate segmentation and detection of tumor in multimodal brain MRI and this research work is focused to improve automatic segmentation results. This work analyses the segmentation performance of existing stateof-art method improved Fuzzy C-Means Clustering (FCMC) method and marker controlled Watershed method and this research work proposed method to amalgamated segmentation results of improved Fuzzy CMeans Clustering (FCMC) method and marker controlled Watershed method to carry out accurate brain tumor detection and enhance the segmentation results. The performance of proposed method is evaluated with assorted performance metric, viz., Segmentation accuracy, Sensitivity and Specificity. The comparative performance of the Proposed Method, FCMC Method and Watershed method is demonstrated on real and benchmark multimodal brain MRI datasets, viz. FLAIR MRI, T1 MRI, MRI and T2 MRI and the experimental results of the proposed method exhibits better results for segmentation and detection of tumor in multimodal brain MR images.
\end{abstract}

Index Terms - Brain Tumor, FCMC Method, Watershed Method, Proposed Method, Bilateral Filter, Brain MRI, Multimodal.

\section{INTRODUCTION}

The Magnetic Resonance Image presents the high soft tissue delineation compared to other medical imaging modalities and it's typically used to analyze a variety of diseases in brain and the image segmentation is majorly used in health care system for analysis and diagnoses of various diseases in various medical applications. Segmentation and detection of brain tumor from multimodal brain MR image is a challenging task due to various factors such as brain MR image generated from diverse scanners with diverse configurations and furthermore the brain tumor varies from intensity varies to healthy tissues and with their shape, size and location is specific to patient.

With the considerable advance in the brain MRI methods have exposed the broad possibilities of brain anatomy analysis based on multimodal Brain MR Images.

The MRI imaging is typically used in the various biomedical applications to diagnose a variety of diseases', to visualize the inner formation of the brain and to detect tumor. MRI technique detects and presents the extreme variations in the soft tissues as compared to other medical imaging techniques. Multimodal Brain MR Images presents the noninvasive broad visualization of internal anatomical understanding of the brain. In order to improve the accuracy for detection of tumor in multimodal Brain MR Images, the imaging techniques have extended the quality of the Brain MR images. Hence the exploration of this complex and high quality Brain MR Images became the major tedious task for the technicians [1]. Moreover, due to the human intervention the investigations are bound to be erroneous. Also these manual analyses are a lot of time-consuming and restricted in discovering of tumor in multimodal Brain MR Images as compared with the computerized methods [1].

Fuzzy c-means clustering (FCMC) is most accepted method under unsupervised technique which has been effectively used in many applications and several areas like clustering, segmentation, etc. but a few limitations are present for traditional FCMC Method. Hence, over last few decades, some improvements have been proposed to FCMC method and only FCMC method is not enough to obtain a better segmentation and detection of tumor in multimodal brain MR images.

The typically used method for analysis of MR images is segmentation based imaging in clinical analysis. Image segmentation is usually used for measuring and visualizing the brain's anatomical structures, for analyzing brain changes, for delineating pathological regions, and for surgical planning and image-guided 
interventions. The limitations identified from the study exhibits various segmentation techniques are restricted in generating high accuracy and mostly focused in brain tumor detection. The recent researches also fail to achieve the supreme accuracy [2]. Multimodal MRI is mainly used for analysis and detection of brain disorder. In multimodal brain MR images have a variety of series such as T1 MR Image, T2 MRI and FLAIR MR Image. This work presents the detection of tumor in multimodal Brain MR Image.

Over the last few years a various literate review on performance evaluation of the image segmentation methods have been established and the most accepted performance measure methods are Segmentation accuracy, Sensitivity, Precision, Similarity index and Specificity. Sensitivity is defined as the percentage of patients appropriately identified with brain tumor, whereas specificity is the percentage of patients could not appropriately identify with tumor. In this research work, the performance of proposed method is evaluated with various performance measures, viz., Segmentation accuracy, Sensitivity and Specificity on real multimodal brain MRI and benchmark multimodal brain MRI datasets.

The rest of the paper is organized as follows, in Section II this paper discuss about the Existing techniques for detection of tumor in multimodal Brain MR Images, in Section III this paper discuss about Proposed Method for Detection of Tumor in multimodal Brain Magnetic Resonance Images, in Section IV presents the results tested on multiple multimodal MRI datasets and discussion about results and in Section $\mathrm{V}$ presents the conclusion.

\section{RELATED WORKS}

The Automatic segmentation [2][3][4][5][6] and detection of tumor in Brain MR Image is challenging due to various limitations such as acquired MR image complexity and properties, pathology, extraction of brain image and tumor boundaries from its background image and MR image analysis based on intensity. The related research works have been proposed various state-of-art brain tumor segmentation techniques and methods in literature for analysis for detecting medical disorders. The segmentation methods of brain MR image is mainly divided into two i.e Unsupervised segmentation methods and supervised segmentation methods. Some of the unsupervised techniques are presented, viz., Atlas based, Rule based, Statistical based, Edge based, Texture based and Cluster based and Deformable models is best example for supervised segmentation method A broad range of automatic segmentation techniques are been deployed to detect the brain tumors from the multimodal MR images.

Some of the state-of-art methods [2][3][4][5] [6][13][14] are compared here [Table - 1].Hence with this understanding, the future direction is clear to have accurate and automatic segmentation method for better segmentation and detection of brain tumor in multimodal brain MR images .Hence with this understanding, the future direction is clear to have accurate and automatic segmentation method for better segmentation and detection of brain tumor in multimodal brain MR images.

Table 1. Analysis of Related Methods and Techniques for detection of brain tumor

\begin{tabular}{|c|c|}
\hline $\begin{array}{l}\text { Existing Methods and } \\
\text { Techniques for } \\
\text { detection of brain } \\
\text { tumor }\end{array}$ & $\begin{array}{l}\text { Analyses of Related Methods and } \\
\text { Techniques }\end{array}$ \\
\hline $\begin{array}{l}\text { Watershed } \\
\text { Segmentation } \\
\text { and } \\
\text { Morphological } \\
\text { Operation }\end{array}$ & $\begin{array}{l}\text { Determines the abnormality presence, fully } \\
\text { automatic algorithm, no prior information or } \\
\text { training process needed. The Medium } \\
\text { accuracy of the detection of brain anomalies } \\
\text { for MR Image. Medium Accuracy and } \\
\text { Medium Complexity. Its complex and } \\
\text { tedious process for the detection of brain } \\
\text { anomalies for MR Image. }\end{array}$ \\
\hline K-Mean Technique & $\begin{array}{l}\text { K-means algorithm presents } K \text { centers for } \\
\text { each cluster and it is quick, strong and } \\
\text { simple. High Accuracy and High } \\
\text { Complexity. It presents superior result as } \\
\text { dataset are well separated from each other. }\end{array}$ \\
\hline $\begin{array}{l}\text { Gaussian Mixture } \\
\text { Technique }\end{array}$ & $\begin{array}{l}\text { It gives model for density modeling and } \\
\text { classification. Medium Accuracy and } \\
\text { Medium Complexity }\end{array}$ \\
\hline $\begin{array}{l}\text { Expectation } \\
\text { Maximization-- } \\
\text { Gaussian Mixture } \\
\text { Technique }\end{array}$ & $\begin{array}{l}\text { GMM is An automated algorithm and GMM } \\
\text { algorithm used for segmentation of low- } \\
\text { contrast, noisy MRI of the brain. } \\
\text { Gaussian Mixture Models (GMMs) and EM } \\
\text { is short of taking the spatial information and } \\
\text { uncertainty of data into consideration and it } \\
\text { presents the less accuracy. }\end{array}$ \\
\hline $\begin{array}{l}\text { Support Vector } \\
\text { Mechanism }\end{array}$ & $\begin{array}{l}\text { The Support Vector Machine method is } \\
\text { widely used for high generalization } \\
\text { performance, in particular while the } \\
\text { dimension of the feature space is very high. } \\
\text { This method gives high Accuracy and Low } \\
\text { Complexity for specific MRI datasets. }\end{array}$ \\
\hline
\end{tabular}

Thus this work focus on automatic and accurate of the segmentation and detection of tumor in multimodal Brain MRI and also presents the quantitative evaluation of the performance of Proposed Method over the existing stateof-art Fuzzy C-Means Clustering (FCMC) and Watershed method for the detection of tumor in multimodal Brain MR Images.

\section{PROPSED METHOD}

The primary objective behind this research work is the accurate segmentation and detection of tumor in multimodal Brain MR Images, viz., FLAIR MRI, T1 MRI and T2 MRI. To achieve the objective of MR image segmentation, this work proposed a method that merges the results of most established improved Fuzzy C-Means Clustering method and marker controlled watershed method. The FCMC and Watershed segmentation methods are applied on the given MR image and then acquired results are optimally unified using proposed method to achieve the higher automatic segmentation accuracy.

The visual representation of brain imaging results in multimodal images such as T1 MRI, T2 MRI and FLAIR MRI. Hence, this work focused on improvement of 
automatic segmentation accuracy and detection of tumor in multimodal brain MR Image's with proposed method. The block diagram for this work is shown in [Figure - 1] and this work is equipped with the bilateral filter [12] for smooth edges and for the better segmentation of multimodal brain MR Images and detection of brain tumor. The core framework of presented approach has been demonstrated in [Figure -4].

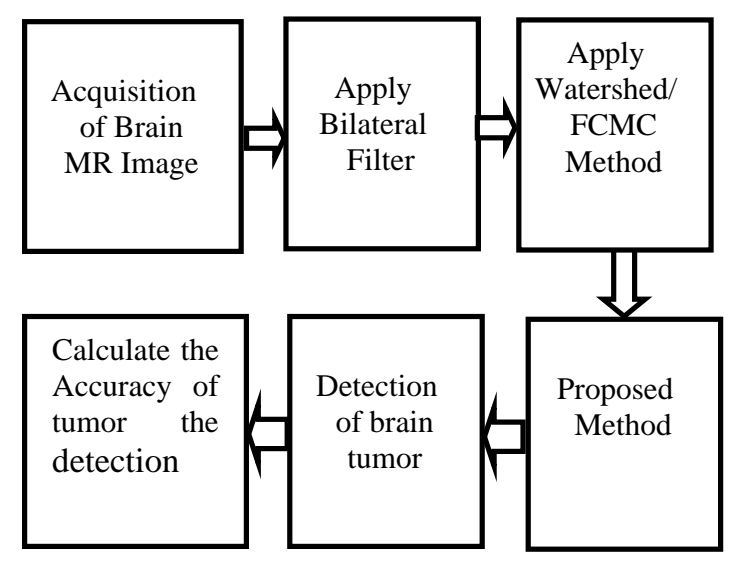

Fig.1. Block diagram of the work.

\section{A.Proposed Framework}

Development of best possible unified framework to amalgamation of segmented regions of existing state-ofthe-art brain tumor segmentation methods Fuzzy CMeans Clustering (FCMC) method and Watershed method.

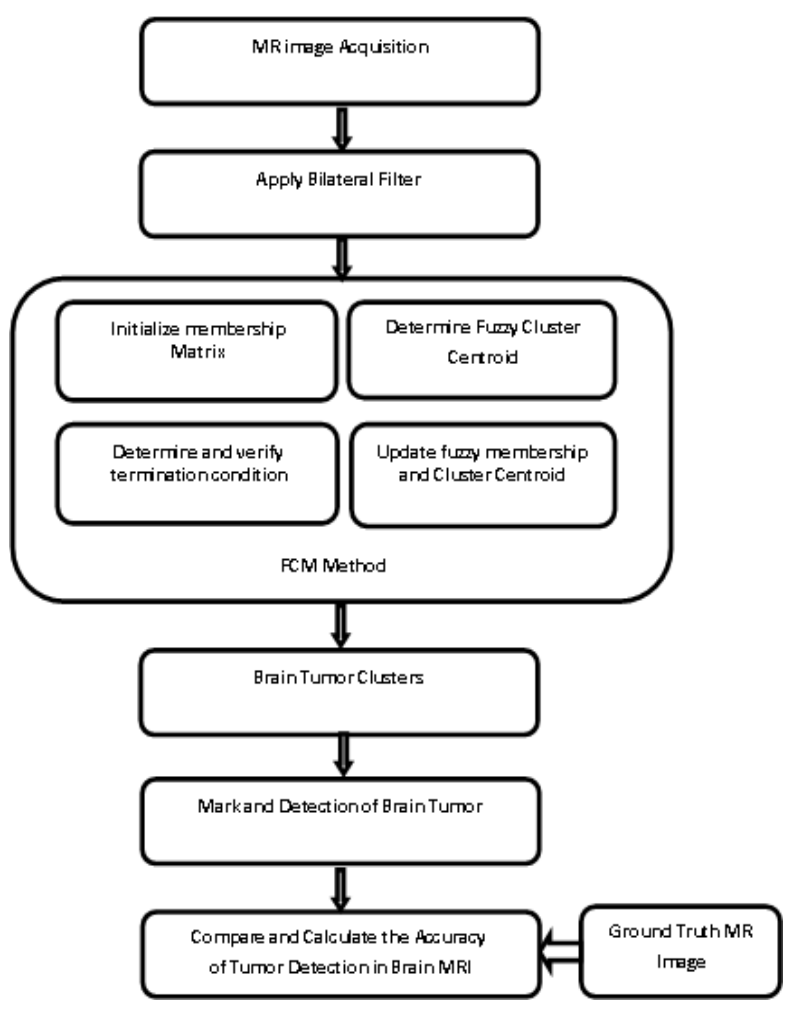

Fig.2. FCMC Method Framework for automatic segmentation and detection of Tumor in multimodal Brain MR Images.
FCMC method [8] [9] [10] [13] is unsupervised automatic segmentation methods and it plays vital role for segmentation and detection of anomalies in brain MR images and it accelerate the MRI segmentation process and FCMC method frame works is shown in[Figure-2]. The drawback of FCMC method is the less segmentation accuracy for the detection of anomalies in multimodal brain MR images.

The watershed method [5] [6] [7] [15] is described as morphological gradient based segmentation for this work and the minimal watershed method is illustrated in [Figure - 3].The objective of the watershed algorithm [1][2] [4][13] is to improve the accuracy of the image segmentation, Watershed algorithm is depicted in [Figure-3].

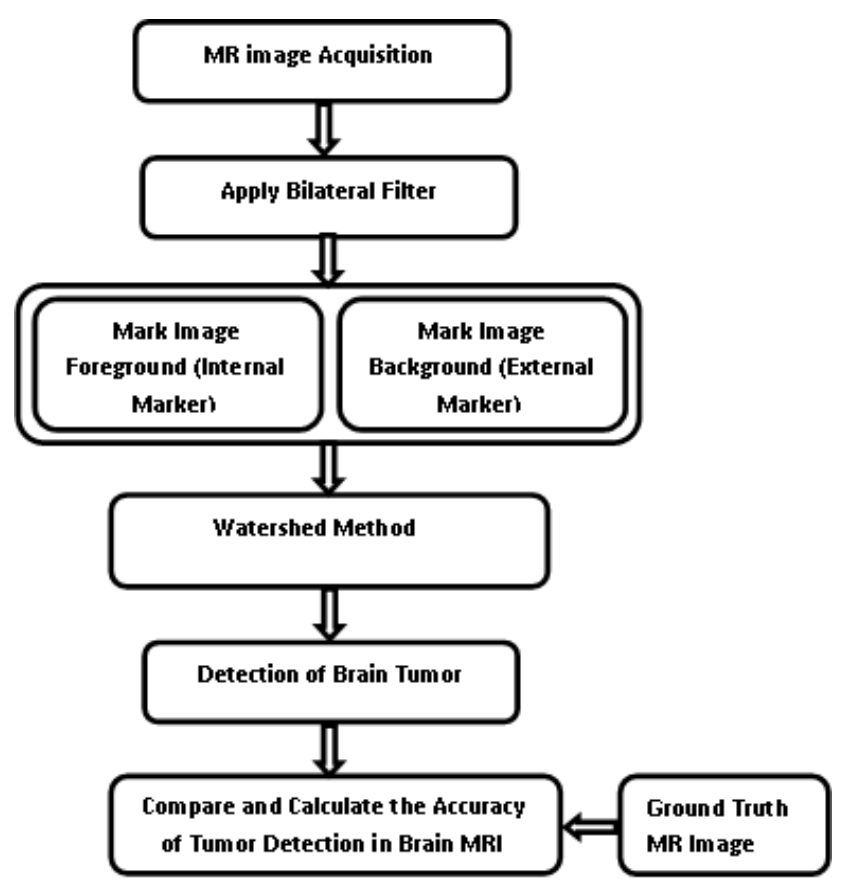

Fig.3. Watershed Method Framework for automatic segmentation and detection of Tumor in multimodal Brain MR Images.

The existing most accepted brain tumor segmentation methods Fuzzy C-Means Clustering (FCMC) method and Watershed method (WM) exhibits the limited segmentation accuracy for the detection of tumor in multimodal brain MR images. Proposed Framework [Figure -4 ] for automatic segmentation and detection of tumor in multimodal brain MR Image overcome the existing limitations of segmentation accuracy.

The major focus of the proposed method is to enhance the segmentation results of tumor detection in multimodal brain MRI by finest merging [11] of segmented regions of Watershed method and FCMC methods. The Proposed method is equipped with the bilateral filter[12] to improve the MRI edges for better segmentation.

\section{B.Algorithm for Proposed Method}

In the proposed method, the finest sets of segments are uniquely divided and similar segmented regions results are merged and finally whole regions are identified and 
considered with the brain regions detected with tumor. The algorithm for proposed method is illustrated below.

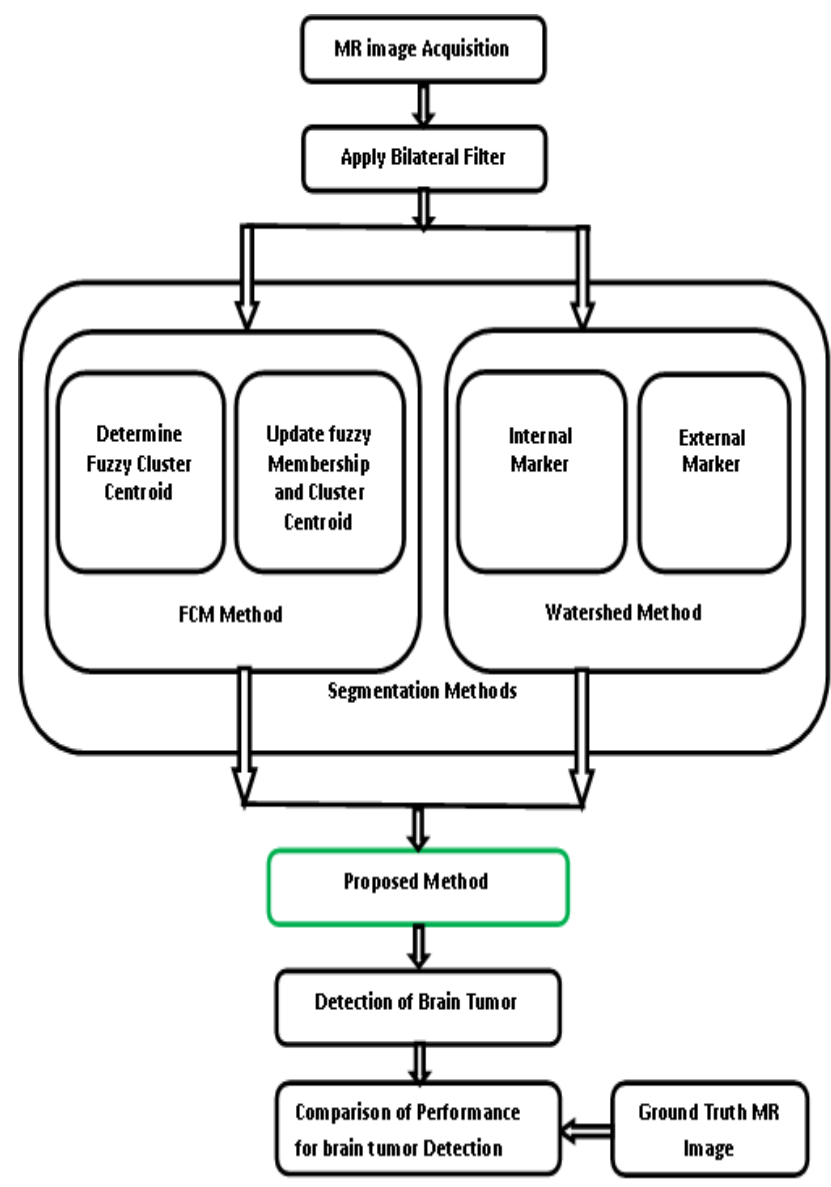

Fig.4. Proposed Method Framework for automatic segmentation and detection of Tumor in multimodal Brain MR Images.

Input: wm_seg and fcmc_seg are the segmentation results from Watershed and FCMC method respectively

Step1 Calculate the number of regions for wm_seg and fcmc_seg and store in $\mathrm{x}$ and $\mathrm{y}$ respectively.

$$
w m_{-} s \mathrm{~g} \rightarrow x, \mathrm{fcmc}_{-} \mathrm{seg} \rightarrow y
$$

Step2 Copy wm_seg and fcmc_seg into SegtList1 [x] and SegList2 [y], Repeat Till $\mathrm{x}+\mathrm{y}$, where $\mathrm{x}+\mathrm{y}$ denotes the size of the SegList arrays.

$$
\begin{aligned}
& \text { SegList } 1[x] \leftarrow w m_{-} \text {seg, } \\
& \text { SegList } 2[y] \leftarrow f c m c_{-} \text {seg. }
\end{aligned}
$$

Step3 Compare All SegList1[x] and SegList2[y] to find the unique region.

$$
\left[\operatorname{SegList}_{1}[x]\right]_{x_{i} \neq x_{j}},\left[\operatorname{SegLiSt}_{2}[y]\right]_{y_{i} \neq y_{j}}
$$

Step4 If SegtList1 [x] and SegList2 [y] are unique, then Store the segmented regions into the SegList1, SegList2 from wm_seg and fcmc_seg respectively.

$$
\begin{aligned}
& {[w m-\operatorname{seg}]_{x_{i} \neq y_{j}} \rightarrow \text { SegList }_{1}} \\
& {[f \mathrm{cmc}-\operatorname{seg}]_{x_{i} \neq y_{j}} \rightarrow \text { SegList }_{2} .}
\end{aligned}
$$

Step5 Compare each Segment from SegtList1 and SegtList2 to find the similar regions using similarity index. If the regions are nearing neighbors, then combine the regions.

Step6 Determine the total regions from Step4 and Step5. Mark the regions with anomalies.

\section{C.Flowchart for Proposed Method:-}

The proposed method for detection of tumor in multimodal brain MRI procedure is described as follows:

1. Proposed method takes input of segmented regions of FCMC method and Watershed method.

2. It determines and store total number of segmented brain MRI regions from FCMC method and Watershed method.

3. Identify and store the unique segmented regions from FCMC method and Watershed method.

4. Identify the similar segmented regions from FCMC method and Watershed method. If the regions are nearing neighbors and then merges the regions.

5. Determine the whole regions from similar and unique brain MRI regions and mark the regions with tumor.

The flowchart of the proposed method is depicted in [Figure-5].

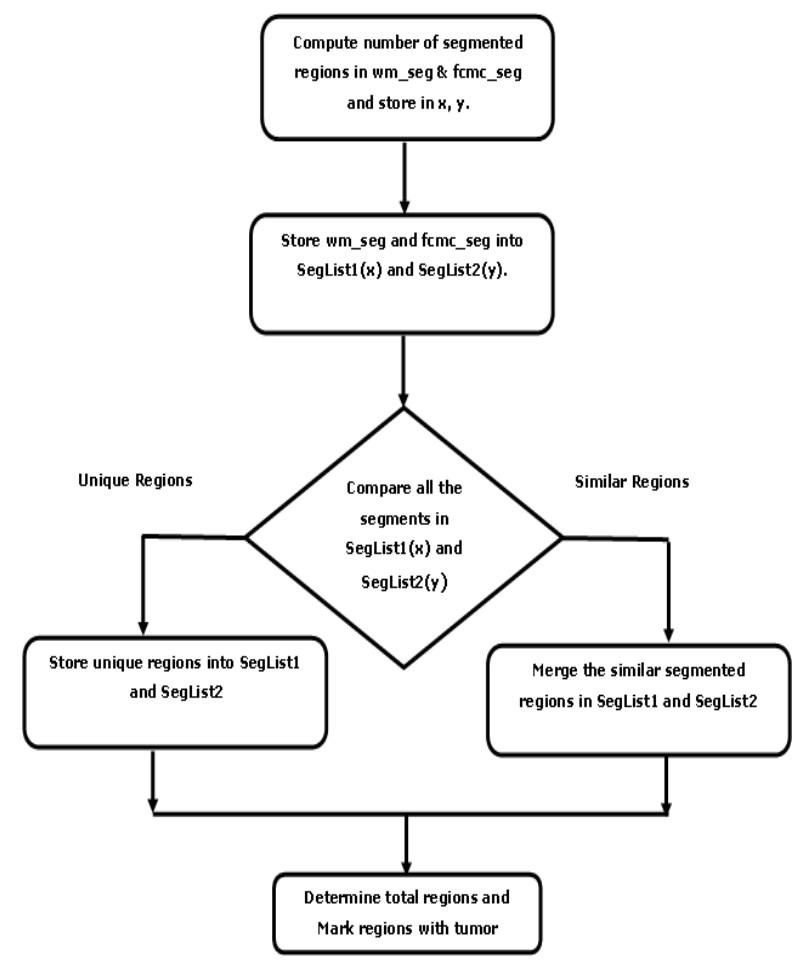

Fig.5. Flowchart for Proposed Method. 


\section{RESULTS AND DISCUSSIONS}

In order to demonstrate the results and theoretical construction presented in this work, we provide the MATLAB implementation of the framework to test the visual advantages of FCMC method, Watershed method and Proposed Method for the detection of tumor in multimodal brain MR images. In this research work, the performance of proposed method is evaluated with following metrics like Segmentation accuracy, Sensitivity and Specificity on real multimodal brain MRI and benchmark multimodal brain MRI datasets.

$$
\begin{gathered}
\begin{array}{c}
\text { Segmentation Accuracy }(\%)= \\
\text { TruePositive +TrueNegative }
\end{array} \\
\frac{\text { Sensitivity }(\%)=}{\text { TruePositive + TrueNegative + FalsePositive + FalseNegative }} * 100 \\
\frac{\text { TruePositive }}{\text { TruePositve + FalseNegative }} * 100 \\
\text { Specificity }(\%)= \\
\frac{\text { TrueNegative }}{\text { TrueNegative + FalsePositive }} * 100
\end{gathered}
$$

The segmentation results of the real multimodal brain MRI is shown in [Figure-6] and the segmentation results of the benchmark datasets is shown in [Figure-7]. The

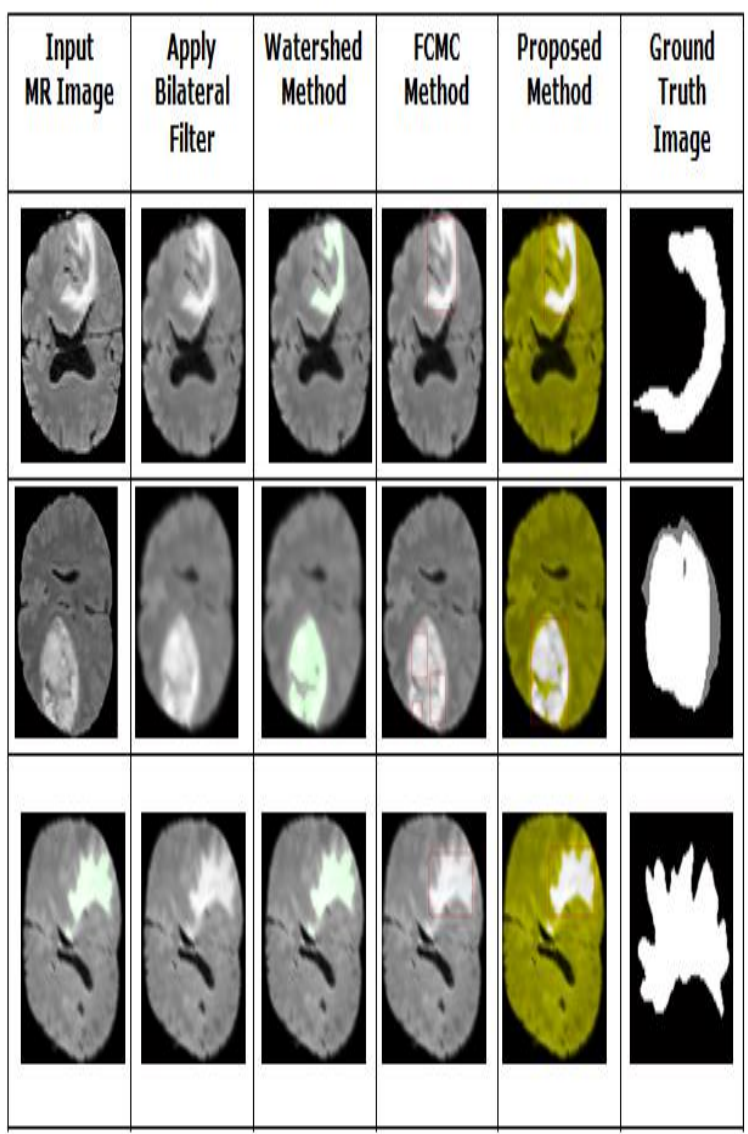

Fig.6. Automatic Segmentation and Detection of tumor in real brain MR Image. accuracy of FCMC Method, Watershed method and Proposed Method is measured by finding the comparison between the tumor extracted from input multimodal Brain MR Images and the ground truth image of the parallel input image that is presented in the dataset. we have analyzed FLAIR MRI [Table-2], T1 MRI [Table-3] and T2 MRI [Table-4] using performance metrics like the segmentation accuracy, sensitivity and Specificity with equation (5), (6) \& (7) using FCMC method, Watershed method and Proposed method for the detection of tumor in multimodal brain MR Images. The multimodal brain MRI benchmark datasets contained the brain T1 MRI, T2 MRI and FLAIR MRI images along with their ground truth image.

The comparative accuracy for FCMC, Watershed and Proposed Method is shown in [Table - 2] for three patient's multimodal brain MRI such as FLAR, T1 and $\mathrm{T} 2$.

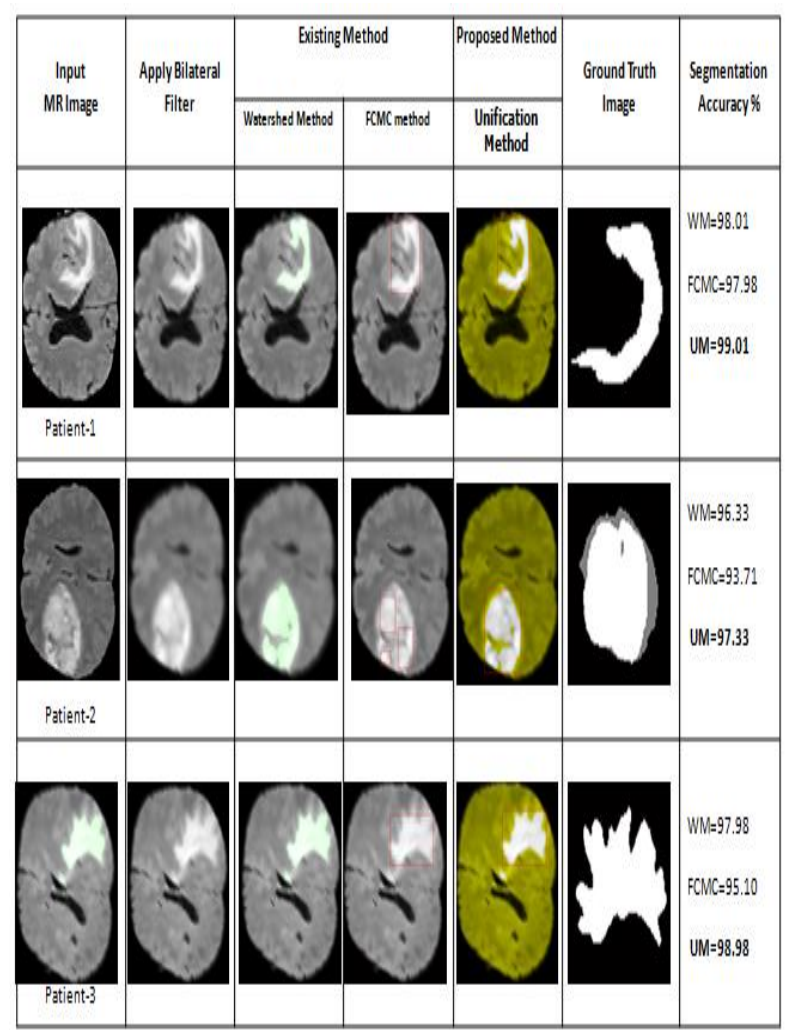

Fig.7. Automatic Segmentation and Detection of brain tumor using FCMC, Watershed and Proposed Method.

The input multimodal Brain MRI dataset is segmented and performance evaluated with segmentation accuracy, sensitivity and Specificity with equation (5), (6) \& (7) for FCMC Method, Watershed method and Proposed Method and analysis results are depicted in [Table-2] [Table-3] [Table-4].

The performance analysis of segmentation results of FCMC Method, Watershed method and Proposed Method are evaluated with following metric segmentation accuracy, sensitivity and specificity on FLAIR Brain MRI dataset and experimental results are depicted in [Table-2]. 
Table 2. Analysis of FLAIR MRI

\begin{tabular}{|c|c|c|c|c|}
\hline $\begin{array}{c}\text { Input MR } \\
\text { Image }\end{array}$ & $\begin{array}{c}\text { Performance } \\
\text { Metrics }\end{array}$ & $\begin{array}{c}\text { FCMC } \\
\text { Method }\end{array}$ & $\begin{array}{c}\text { Watershed } \\
\text { Method }\end{array}$ & $\begin{array}{c}\text { Proposed } \\
\text { Method }\end{array}$ \\
\hline & Accuracy (\%) & 97.39 & 98.14 & 98.98 \\
& Sensitivity (\%) & 80.32 & 69.73 & 69.66 \\
Patient-1 & Specificity (\%) & 97.78 & 98.86 & 99.86 \\
\hline & Accuracy (\%) & 96.90 & 98.30 & 99.18 \\
& Sensitivity (\%) & 94.73 & 93.59 & 93.40 \\
Patient-2 & Specificity (\%) & 97.02 & 98.52 & 99.55 \\
\hline & Accuracy (\%) & 93.741 & 97.20 & 97.97 \\
& Sensitivity (\%) & 95.22 & 95.34 & 95.25 \\
Patient-3 & Specificity (\%) & 93.71 & 98.10 & 98.10 \\
\hline & Accuracy (\%) & 95.83 & 97.26 & 98.05 \\
& Sensitivity (\%) & 89.51 & 90.78 & 90.78 \\
Patient-4 & Specificity (\%) & 95.87 & 98.13 & 98.13 \\
\hline & Accuracy (\%) & 94.34 & 95.99 & 96.78 \\
& Sensitivity (\%) & 96.83 & 97.70 & 97.71 \\
Patient-5 & Specificity (\%) & 94.30 & 96.75 & 96.76 \\
\hline
\end{tabular}

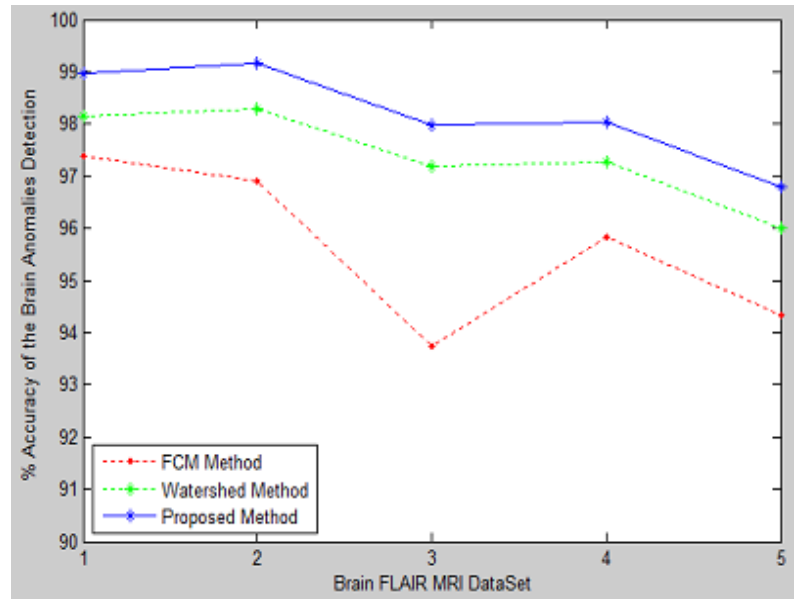

Fig.8. Comparison of segmentation Accuracy for brain FLAIR MRI

The comparative performance of the segmentation accuracy of FCMC Method, Watershed Method and Proposed Method on FLAIR MR Image is shown in [Figure-8].

Table 3. Aanalysis of T1 MRI

\begin{tabular}{|c|l|c|c|c|}
\hline $\begin{array}{c}\text { Input MR } \\
\text { Image }\end{array}$ & $\begin{array}{c}\text { Performance } \\
\text { Metrics }\end{array}$ & $\begin{array}{c}\text { FCMC } \\
\text { Method }\end{array}$ & $\begin{array}{c}\text { Watershed } \\
\text { Method }\end{array}$ & $\begin{array}{c}\text { Proposed } \\
\text { Method }\end{array}$ \\
\hline & Accuracy (\%) & 96.28 & 96.62 & 97.27 \\
& Sensitivity (\%) & 81.21 & 76.12 & 78.65 \\
Patient-1 & Specificity (\%) & 96.43 & 96.33 & 97.49 \\
\hline & Accuracy (\%) & 94.86 & 96.14 & 97.23 \\
& Sensitivity (\%) & 90.13 & 91.55 & 91.65 \\
Patient-2 & Specificity (\%) & 93.15 & 97.23 & 98.67 \\
\hline & Accuracy (\%) & 91.43 & 94.26 & 95.57 \\
& Sensitivity (\%) & 93.15 & 91.38 & 91.49 \\
Patient-3 & Specificity (\%) & 92.54 & 96.20 & 96.40 \\
\hline & Accuracy (\%) & 93.46 & 95.56 & 97.15 \\
& Sensitivity (\%) & 90.78 & 91.72 & 89.75 \\
Patient-4 & Specificity (\%) & 94.65 & 96.43 & 97.33 \\
\hline & Accuracy (\%) & 93.43 & 94.17 & 95.48 \\
& Sensitivity (\%) & 84.56 & 73.33 & 79.32 \\
Patient-5 & Specificity (\%) & 94.67 & 96.61 & 97.12 \\
\hline
\end{tabular}

The performance analysis of segmentation results of FCMC Method, Watershed method and Proposed Method are evaluated with following metric segmentation accuracy, sensitivity and specificity on T1 Brain MRI dataset and experimental results are depicted in [Table-3].

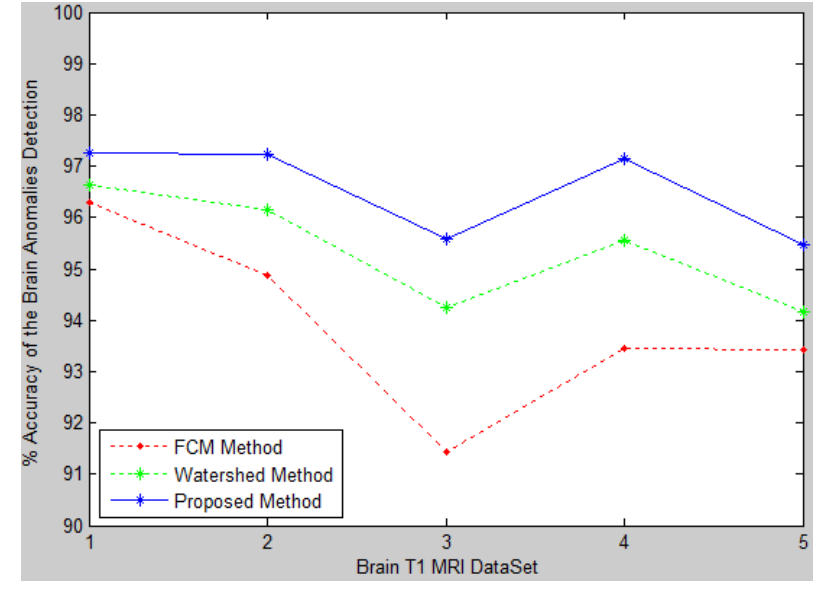

Fig.9. Comparison of segmentation Accuracy for brain T1 MRI.

The comparative performance of the segmentation accuracy of FCMC Method, Watershed Method and Proposed Method on T1 MR Image is shown in [Figure9].

Table 4. Analysis of T2 MRI

\begin{tabular}{|c|l|c|c|c|}
\hline $\begin{array}{c}\text { Input MR } \\
\text { Image }\end{array}$ & $\begin{array}{c}\text { Performance } \\
\text { Metrics }\end{array}$ & $\begin{array}{c}\text { FCMC } \\
\text { Method }\end{array}$ & $\begin{array}{c}\text { Watershed } \\
\text { Method }\end{array}$ & $\begin{array}{c}\text { Proposed } \\
\text { Method }\end{array}$ \\
\hline & Accuracy (\%) & 93.43 & 94.34 & 95.81 \\
& Sensitivity (\%) & 78.32 & 77.45 & 79.24 \\
Patient-1 & Specificity (\%) & 95.78 & 95.81 & 95.41 \\
\hline & Accuracy (\%) & 91.12 & 93.81 & 94.56 \\
& Sensitivity (\%) & 83.35 & 81.32 & 81.25 \\
Patient-2 & Specificity (\%) & 93.71 & 94.61 & 95.66 \\
\hline & Accuracy (\%) & 89.36 & 91.29 & 92.37 \\
& Sensitivity (\%) & 84.81 & 83.81 & 82.74 \\
Patient-3 & Specificity (\%) & 91.14 & 93.21 & 93.90 \\
\hline & Accuracy (\%) & 90.62 & 92.14 & 93.25 \\
& Sensitivity (\%) & 88.35 & 85.16 & 81.61 \\
\hline \multirow{5}{*}{ Patient-4 } & Specificity (\%) & 92.67 & 93.31 & 94.27 \\
\hline & Accuracy (\%) & 91.42 & 93.14 & 94.31 \\
& Sensitivity (\%) & 82.81 & 78.18 & 83.88 \\
& Specificity (\%) & 92.71 & 94.31 & 94.25 \\
\hline
\end{tabular}

The performance analysis of segmentation results of FCMC Method, Watershed method and Proposed Method are evaluated with following metric segmentation accuracy, sensitivity and specificity on T2 Brain MRI dataset and experimental results are depicted in [Table-4].

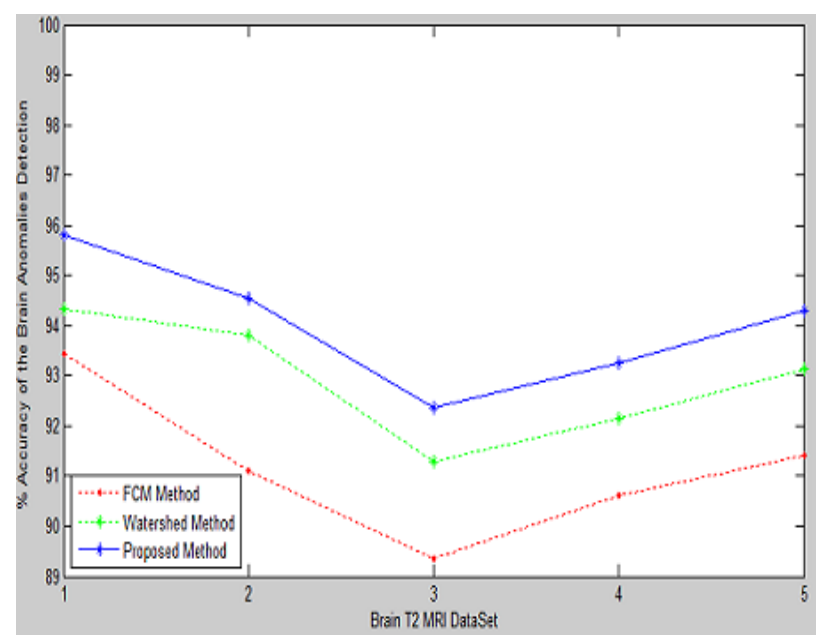

Fig.10. Comparison of segmentation Accuracy for brain T2 MRI. 
The comparative performance of the segmentation accuracy of FCMC Method, Watershed Method and Proposed Method on T2 MR Image is shown in [Figure$10]$.

This research work exhibits the comparative performance of the segmentation accuracy of FCMC Method, Watershed Method and Proposed Method on multimodal brain MR images, viz,. FLAIR MRI, T1 MRI and T2 MRI are shown in [Figure-9] [Figure-10] and [Figure-11]. The proposed method in the experimental results clearly demonstrated improved segmentation results for multimodal brain MR images.

\section{CONCLUSION}

The research work majorly focused on the performance improvement of automatic segmentation and accurate detection of tumor in multimodal Brain MR Images and also presented extensive analysis of the automatic segmentation methods for detection of the brain tumor in multimodal Brain MR Images. The state-of-art methods for brain tissue segmentation are developed and demonstrated on multimodal brain MR images. The performance of proposed method has been evaluated on real and benchmark multimodal brain MRI datasets, viz. FLAIR MRI, T1 MRI, MRI and T2 MRI with following performance metric, viz., Segmentation accuracy, Sensitivity and Specificity. The proposed method in this research work demonstrated improved performance for detection of the brain tumor in multimodal Brain MR Images. The work also presented the relative analysis of Proposed Method, FCMC Method and Watershed method for segmentation of multimodal Brain MRI segmentation and detection of brain tumor. With the final outcome of performance development, the proposed method is efficient for automatic segmentation and detection of tumor regions in Brain MR Images. This research work extends the possibilities of better segmentation and accurate detection of brain tumor in multimodal MR Images

\section{REFERENCES}

[1] N Van . Porz, "Multi-modalodal glioblastoma segmentation: Man versus machine", PLOS ONE, vol. 9, pp. e96873, 2014.

[2] S. Bauer, R. Wiest, L.-P. Nolte and M. Reyes, "A survey of MRI-based medical image analysis for brain tumor studies", Phys. Med. Biol., vol. 58, no. 13, pp. R97-R129, 2013.

[3] L. Weizman, "Automatic segmentation, internal classification, and follow-up of optic pathway gliomas in MRI", Med. Image Anal., vol. 16, no. 1, pp. 177-188, 2012.

[4] S. Ahmed, K. M. Iftekharuddin and A. Vossough, "Efficacy of texture, shape, and intensity feature fusion for posterior-fossa tumor segmentation in MRI", IEEE Trans. Inf. Technol. Biomed., vol. 15, no. 2, pp. 206-213, 2011.

[5] Jin Liu, Min Li, Jianxin Wang, Fangxiang Wu, Tianming Liu, and Yi Pan, "A Survey of MRI-Based Brain Tumor Segmentation Methods", TSINGHUA SCIENCE AND TECHNOLOGY, Volume 19, Number 6, December 2014.
[6] J. B. T. M. Roerdink and A. Meijster, "The watershed transform: Definitions, lgorithms and parallelization strategies," Fundamenta Informaticae,vol. 41, pp. 187-228, 2000.

[7] Gang Li , "Improved watershed segmentation with optimal scale based on ordered dither halftone and mutual information”, Page(s) 296 - 300, Computer Science and Information Technology (ICCSIT), 2010, 3rd IEEE International Conference, 9-11 July 2011.

[8] Benson. C. C, Deepa V, Lajish V. L and Kumar Rajamani, "Brain Tumor Segmentation from MR Brain Images using Improved Fuzzy c-Means Clustering and Watershed Algorithm", Intl. Conference on Advances in Computing, Communications and Informatics (ICACCI), Sept. 21-24, 2016, Jaipur, India.

[9] L'aszl'o Szil'agyi,L'aszl'o Lefkovits and Bal'azs Beny'o, "Automatic Brain Tumor Segmentation in Multispectral MRI Volumes Using a Fuzzy c-Means Cascade Algorithm", 12th International Conference on Fuzzy Systems and Knowledge Discovery (FSKD),2015.

[10] G.-C. Lin, W.-J. Wang, C.-C. Kang and C.-M. Wang, "Multispectral mr images segmentation based on fuzzy knowledge and modified seeded region growing", Magnetic Resonance Imaging, vol. 30, no. 2, pp. 230-246, 2012.

[11] NageswaraReddy P, C.P.V.N.J.Mohan Rao, Ch.Satyanarayana, "Optimal Segmentation Framework for Detection of Brain Anomalies", I.J. Engineering and Manufacturing, 2016, 6, 26-37, Published Online November 2016 in MECS.

[12] Devanand Bhonsle, Vivek Chandra , G.R. Sinha3 , "Medical Image Denoising Using Bilateral Filter", I.J. Image, Graphics and Signal Processing, 2012, 6, 36-43. Published Online July 2012 in MECS.

[13] Sanjay Kumar, Santosh Kumar Ray, Peeyush Tewari,, A "Hybrid Approach for Image Segmentation Using Fuzzy Clustering and Level Set Method", I.J. Image, Graphics and Signal Processing, 2012, 6, 1-7, Published Online July 2012 in MECS.

[14] Kalaiselvi T and Nagaraja P, "An Automatic Segmentation of Brain Tumor from MRI Scans through Wavelet Transformations", I.J. Image, Graphics and Signal Processing, 2016, 11, 59-65, Published Online November 2016 in MECS.

[15] Anam Mustaqeem, Ali Javed, Tehseen Fatima, "An Efficient Brain Tumor Detection Algorithm Using Watershed \& Thresholding Based Segmentation", I.J. Image, Graphics and Signal Processing, 2012, 10, 34-39, Published Online September 2012 in MECS.

\section{Authors' Profiles}

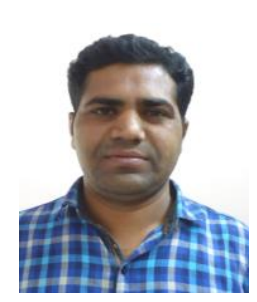

K.Bhima received his B.Tech degree in Information Technology from R V R \& J C College of Engineering, Guntur in 2004 and M.Tech degrees in Software Engineering from NIT Allahabad, India in 2006 and currently working as Associate Professor in Department of Computer Science and Engineering, B.V. Raju Institute of Technology, Narsapur, Medak, Telangana State, India. He his is currently working toward the Ph.D. degree in the Department of Computer Science and Engineering from 
Jawaharlal Nehru Technological University, Hyderabad. He has 12 years of teaching and research experiences in engineering colleges. His current research and teaching interests include Image Processing, Network Security and Software Engineering. He has been presented and published over 12 research papers in National, international Conferences and Journals.

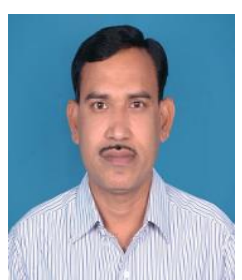

Dr. A. Jagan received his B.E degree in Electronic and Communication Engineering from Osmania University, Hyderabad in 1995 and M. Tech degrees in Computer Science and Engineering from JNTU University, Hyderabad, India in 1999. He has been awarded with Ph.D by JNTU, Hyderabad in 2011 for his extensive research work in the field of Image processing and currently working as Professor and HOD in the Department of Computer Science and Engineering, B.V. Raju Institute of Technology, Narsapur, Medak, Telangana State, India.His current research interests include Image Processing, Mobile Communication, information Security, Computer Networks, Distributed and Grid computing. He has been presented and published over 38 research papers in National, international Conferences and Journals.

How to cite this paper: K Bhima, A Jagan,"An Improved Method for Automatic Segmentation and Accurate Detection of Brain Tumor in Multimodal MRI", International Journal of Image, Graphics and Signal Processing(IJIGSP), Vol.9, No.5, pp.1-8, 2017.DOI: 10.5815/ijigsp.2017.05.01 